\title{
Nefrectomía laparoscópica en un programa de donante vivo en la Costa Caribe de Colombia
}

\section{Laparoscopic nephrectomy in a living donor program in the Caribbean region of Colombia}

\author{
Yenny Baez-Suarez ${ }^{1}$ iD, Javier Amaya-Nieto² ${ }^{\text {iD, }}$ Fernando Girón-Luque ${ }^{3}$ iD
}

\begin{abstract}
1. Colombiana de trasplantes. Bogotá, Colombia. Correo: ybaez@colombianadetrasplantes.com - https://orcid.org/0000-0002-0823-507X
2. Colombiana de trasplantes. Bogotá, Colombia. Correo: jamaya@colombianadetrasplantes.com - https://orcid.org/0000-0002-9856-6242

3. Colombiana de trasplantes. Bogotá, Colombia. Correo: fgiron@colombianadetrasplantes.com - https://orcid.org/0000-0001-6307-0848
\end{abstract}

Tipología: Artículo de investigación científica y tecnológica

Para citar este artículo: Baez-Suarez Y, Amaya-Nieto J, Girón-Luque F. Nefrectomía laparoscópica en un programa de donante vivo en la Costa Caribe de Colombia. Duazary. 2020 julio - septiembre; 17(3): 25 - 34. Doi 10.21676/2389783X.3319

Recibido en mayo 24 de 2018

Aceptado en noviembre 13 de 2019

Publicado en línea en abril 18 de 2020

\begin{abstract}
RESUMEN
Palabras clave: Trasplante de riñón; obtención de tejidos y órganos; laparoscopía; nefrectomía

La nefrectomía laparoscópica en donante vivo es la mejor opción en el proceso del trasplante para pacientes con enfermedad renal crónica en estadio cuatro y cinco. El objetivo del artículo es describir la experiencia en el programa de donante vivo de riñón entre el 2013 y el 2018 en Colombiana de Trasplantes en la costa Caribe colombiana. Se realizó un estudio descriptivo que incluyó una muestra consecutiva de 55 donantes vivos de riñón operados por Colombiana de Trasplantes, entre enero de 2013 y enero de 2018; se llevó a cabo el análisis estadístico de las variables relevantes. La media de la edad fue de 41 años. El 57,4\% de los donantes fueron mujeres. El tiempo de cirugía tuvo una media de 1,9 horas. Ninguno de los pacientes falleció en el seguimiento a los seis meses y el promedio de la estancia hospitalaria posterior a la cirugía fue de dos días. Las variables relevantes y los desenlaces de los pacientes son similares a los de otros grupos de trasplantes, sin embargo, hay características específicas que pueden ayudar a mejorar las estrategias en salud en la costa Caribe colombiana.
\end{abstract}

\section{ABSTRACT}

Keywords:

Kidney

transplant;

Tissue and

organ

procurement;

laparoscopy;

nephrectomy.
Laparoscopic nephrectomy in living donor is the best option during the process of kidney transplantation for patients with end-stage chronic renal failure. The aim of this article is to describe the experience in a kidney living donor program since 2013 to 2018 at "Colombiana de Trasplantes" in the Colombian Caribbean region. A descriptive study was performed in which a consecutive sample of 55 living donors operated in "Colombiana de Trasplantes" since January 2013 to January 2018. Statistical analysis was done on the relevant variables. The mean of the age was 41 years. $57.4 \%$ of the living donors were female. Surgical time had mean time of 1.9 hours. None of the donors died after 6 months follow-up and the mean time of postoperative stay was 2 days. Important variables and main outcomes in living donors are similar to what is shown by other groups. However, there are specific characteristics that could help to improve health strategies in the Caribbean Region. 


\section{INTRODUCCIÓN}

La nefrectomía laparoscópica (NL) fue introducida como una alternativa en el protocolo de donante vivo en 1995 después de que Ratner et $a l^{1}$ realizaron por primera vez dicho procedimiento quirúrgico ${ }^{2}$. Posteriormente en 1998, Wolf et al ${ }^{3}$. Agregaron la nefrectomía laparoscópica manoasistida (NLMA) al repertorio de técnicas quirúrgicas acusando menor tiempo en la curva de aprendizaje y de esta manera, mejores resultados quirúrgicos ${ }^{3}$.

La NL y la NLMA se han afianzado a lo largo de las dos últimas décadas como la opción estándar sobre la versión abierta, debido al mejor resultado estético, menores requerimientos analgésicos posquirúrgicos, disminución de la estancia hospitalaria posterior al procedimiento, así como un periodo de convalecencia más corto ${ }^{1,4,5}$. Sin embargo, es de relevancia precisar que la NLMA ha sido asociada a menor tiempo quirúrgico, menor tiempo de isquemia caliente, menor sangrado al permitir compresión directa y a ventajas técnicas como la posibilidad realizar disección digital, así como la posibilidad de extraer el riñón con mayor rapidez. Actualmente existen variantes menos frecuentes de estas dos técnicas como lo son la NLMA retroperitoneal que argumenta un riesgo menor de lesión de estructuras adyacentes y las NL con extracción del riñón por orificios naturales ${ }^{6,7}$. Una cohorte de 507 nefrectomías por laparoscopia via retroperitoneal reporto menor tiempo en isquemia caliente sin requerimiento de manipulación intraperitoneal ${ }^{8}$. La nefrectomía laparoscópica robot-asistida también ha presentado un auge importante, aunque requiere el debido y correcto entrenamiento ${ }^{9}$. Del mismo modo, se están realizando diferentes protocolos peri, intra y posoperatorios para mejorar los resultados de la nefrectomía por laparoscopia como por ejemplo, el uso de anestesia epidural posoperatoria ${ }^{10}$.

Por otro lado, se ha estudiado la costo-efectividad de las diferentes técnicas de nefrectomía, siendo la NLMA más costo-efectiva en términos de calidad de vida bajo el costo total en comparación de la NL y nefrectomía abierta, siendo la nefrectomía laparoscópica robot-asistida superior en $\operatorname{costos}^{11}$.
La nefrectomía por laparoscopia toma vital importancia para el programa de donante vivo el cual mejora la oportunidad de trasplante de los receptores, la sobrevida del injerto y del paciente trasplantado ${ }^{12}$. En Colombia, según lo reportado por Osorio et $a l^{13}$ con información recolectada entre 2008 y 2012 , tan solo un $8,3 \%$ del total de los trasplantes fue de donante vivo, de los cuales la mayoría correspondía a receptores hombres. En el año 2018, el Instituto Nacional de Salud (INS) colombiano reporto 114 trasplantes renales con donante vivo, lo que equivale a un $16,4 \%$ de la actividad trasplantadora renal, la cual lentamente va aumentando ${ }^{14}$. En Latinoamérica, los trasplantes de donante vivo en el año 2014 tampoco superaron el $34 \%$ del total de los trasplantes reportados ${ }^{13}$. En Estados Unidos de América (USA), se reportó un $30,4 \%$ de los trasplantes renales con donante vivo en el año $2018^{15}$. Así, se determina una gran necesidad de aumentar el pool de donantes vivos. Para el aumento de la tasa de donación de donante vivo se han generado estrategias de educación ${ }^{16}$ que permiten informar a los potenciales donantes vivos sobre procedimiento quirúrgico y evitar la negativa por temor a los resultados estéticos, el tiempo de convalecencia posteriores a la cirugía y el dolor que esta puede causarles ${ }^{17}$.

A pesar de que existe información de la situación del trasplante de donante vivo en países que lideran la iniciativa, en Colombia aún hay necesidad de delimitar el contexto para dar claridad a aspectos como las bajas tasas de donación en algunos sitios de la geografía y otras particularidades tanto demográficas como técnicocientíficas; por tal razón, el objetivo de este estudio es describir la experiencia en el programa de donante vivo de riñón en Colombiana de Trasplantes entre el 2013 y el 2018 en la regional de la costa Caribe colombiana.

\section{MATERIALES Y MÉTODOS}

\section{Tipo de estudio}

Estudio descriptivo de corte transversal. 


\section{Población y muestra}

La muestra que se describió fue de 55 pacientes quienes fueron donantes vivos de riñón y que fueron llevados a NLMA por Colombiana de Trasplantes en la ciudad de Barranquilla desde enero de 2013 hasta enero de 2018. Se revisaron las historias clínicas de los pacientes seleccionados por muestreo consecutivo, mayores de edad, que cumplieron con los criterios de selección para el proceso de donante vivo que de forma resumida implican pacientes sin hipertensión, sin diabetes o riesgo de diabetes, sin enfermedades genéticas 0 de otra índole que puedan comprometer la función renal del paciente, así como enfermedades psiquiátricas o condiciones laborales riesgosas con potencial riesgo de lesión renal. También se evalúan la naturaleza de la relación entre el receptor y el donante, buscando una donación completamente altruista. Esta evaluación es realizada por un equipo multidisciplinario que incluye enfermeros, nutricionistas, psicólogos, psiquiatras, nefrólogos, cirujanos de trasplantes y otros especialistas según las particularidades del paciente.

\section{Procedimiento}

Previa autorización del comité de investigaciones de la institución se procedió a recopilar los datos por medio de una consulta en el sistema interno de historia clínica (SISS), los datos obtenidos de dicha búsqueda fueron verificados para garantizar la veracidad de la información brindada por el sistema. La información recolectada corresponde a la experiencia de la regional Atlántico que es una de las seis en las que se divide el país desde la óptica del gobierno central ${ }^{18}$.

Se realizó la revisión de variables demográficas de los pacientes del estudio las cuales permitieron caracterizar la población de donantes vivos en la regional de la costa Caribe colombiana. Por otro lado, se recolectaron variables concernientes al procedimiento quirúrgico como lo son el tiempo quirúrgico expresado en horas, el tiempo de isquemia caliente (tiempo transcurrido desde el clampeo de los vasos renales hasta el enfriamiento del órgano con el líquido de preservación), la pérdida de sangre expresada en mililitros, necesidad de conversión a cirugía abierta, tipo de incisión, multiplicidad de arterias y venas renales, uso de sutura mecánica o ligaclip de polímero no absorbible para el control vascular después de extraer el riñón, complicaciones intraoperatorias y la estancia hospitalaria posterior al procedimiento.

Las variables fueron elegidas pensando en los datos más relevantes para obtener información acerca de la seguridad del procedimiento y enfocadas en los desenlaces del donante.

\section{Análisis de los datos}

Para la recolección de los datos se utilizó una hoja de cálculo de Microsoft Excel la cual fue protegida por medio de una clave que solo tenían los investigadores. Después de depurar la información y verificar la base de datos, se realizó el análisis estadístico de la base de datos por medio del programa de análisis estadístico $\mathrm{R}$ en su versión $\mathrm{R}$ 3.4.9 (Copyright 2007 Free Software Foundation, Inc). Se calcularon las frecuencias absolutas y relativas para describir las variables categóricas $y$, las medias, medianas y rangos para la descripción de las variables numéricas. Después de obtenidos los resultados del estudio, se presentaron en tablas y gráficas correspondientes a cada una de las variables de interés.

\section{Declaración sobre aspectos éticos}

Teniendo en cuenta la resolución 8430 de 1993 del Ministerio de Salud de Colombia y que este estudio fue realizado de forma retrospectiva sobre información documental y no se realizó intervención alguna en la población, es una investigación sin riesgo. Adicionalmente $y$ en cumplimiento de la Declaración de Helsinki, todos los derechos y el bienestar de los individuos nunca fue comprometido dentro del desarrollo de la investigación. También se declara que el manejo de los datos fue realizado de manera profesional, buscando siempre la protección de la confidencialidad de la información tanto en el proceso como en los resultados del estudio.

\section{RESULTADOS}

Después de confirmar que las historias clínicas tenían al menos un $80 \%$ de la información de las variables propuestas, se revisaron 55 historias clínicas. La media de edad de los donantes fue de 41 años y las edades oscilaron entre los 20 y los 61 
años. El $57,4 \%(n=31)$ de los donantes fueron mujeres y el $12,7 \%(n=7)$ de los pacientes fueron donantes vivos relacionados con él receptor. También se encontró que el riñón izquierdo se extrajo en el $87 \%(n=47)$ de los casos (Tabla 1$)$.

Tabla 1. Variables demográficas de la población estudiada.

\begin{tabular}{|c|c|c|}
\hline Variable & Número de pacientes & Porcentaje \\
\hline Numero de nefrectomías laparoscópicas & 55 & \\
\hline Genero (\%) & 24 & 42.6 \\
\hline Masculino & 48 & 87,3 \\
\hline Relación con el receptor (\%) & & \\
\hline Vivo relacionado & 47 & 87,04 \\
\hline Riñón extraído (\%) & & \\
\hline Izquierdo & & \\
\hline
\end{tabular}

En cuanto a lo que tiene que ver con las variables importantes del procedimiento quirúrgico, se encontró que el tiempo quirúrgico promedio expresado en horas fue de $1,9(\mathrm{DE}=0,1)$ y la media del tiempo de isquemia caliente fue de $3,1(D E=0,1)$ minutos. En ninguno de los procedimientos fue necesario convertir a cirugía abierta. En cuanto al sangrado intraoperatorio se encontró un promedio de 80,5 mililitros $(D E=10,1)$ (Figura 1) y la incisión más frecuente fue la transversa suprapúbica, realizada en el $88,8 \%(n=48)$ de los pacientes con respecto a un $11,2 \%(n=6)$ en los que se realizó una incisión mediana infraumbilical.

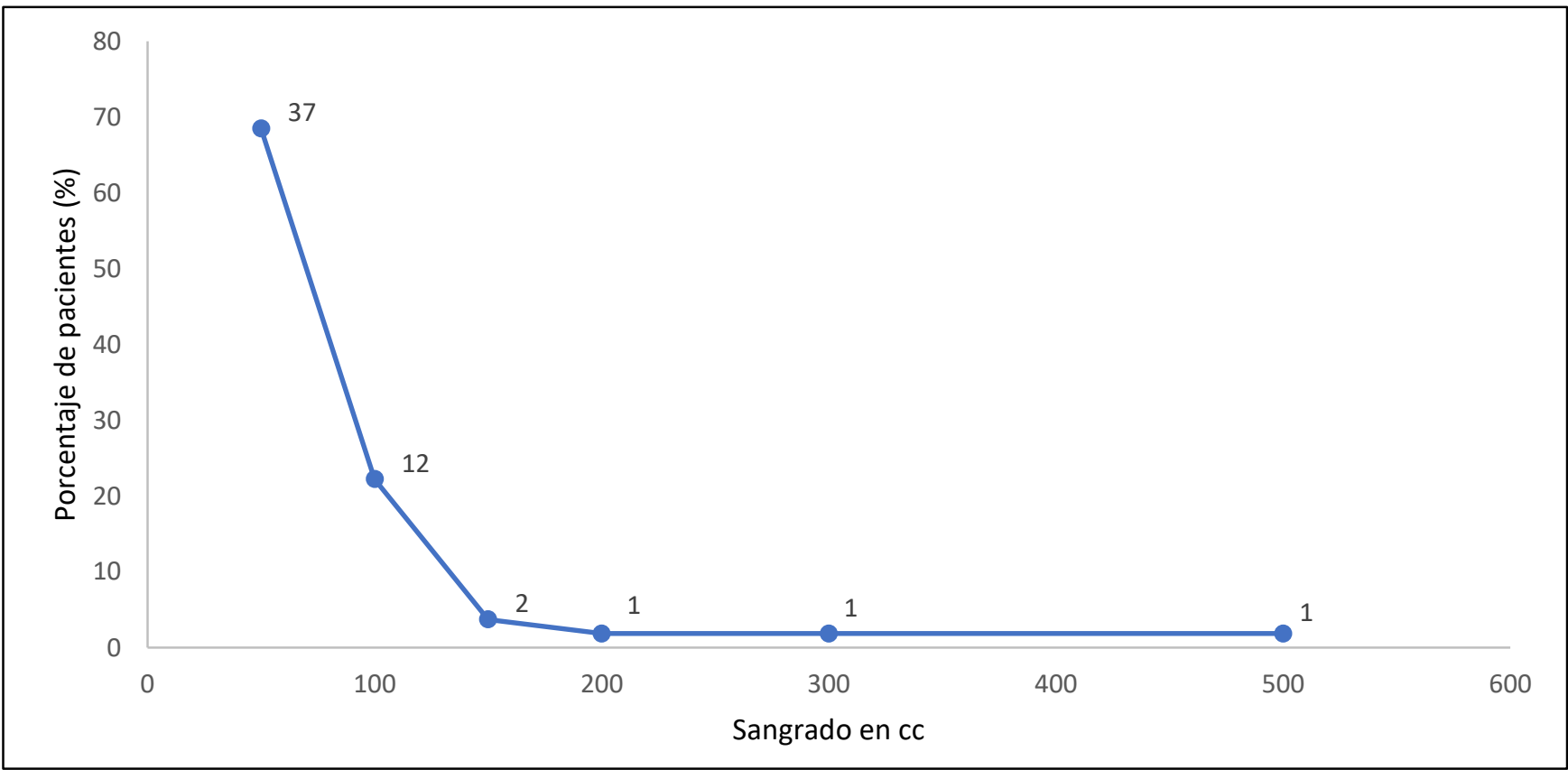

Figura 1. Diagrama de Pareto que muestra el sangrado comparado con el porcentaje y número acumulado de pacientes. La línea naranja muestra el porcentaje acumulado. 
Las variantes anatómicas vasculares renales fue otra de las variables analizadas, encontrando que hubo multiplicidad de arteria renales en mayor proporción ( $22,2 \%$ de los casos, $n=12)$ que de venas renales $(7,4 \%$ de los casos, $n=4)$. Para el control vascular después de la extracción del riñón se utilizaron dos técnicas, la primera y más frecuente fue la doble ligadura con ligaclip de polímero no absorbible, utilizada en el $88,8 \%(n=48)$ de los pacientes y la segunda opción fue la sutura mecánica utilizada en 7 (11,2\%) pacientes en los que se utilizó exclusivamente para venas renales derechas con parche de vena cava inferior (Figura 2). En lo que tiene que ver con las complicaciones intraoperatorias se observó que ninguno desarrolló este tipo de eventos según la clasificación de Clavien para nefrectomía de donante vivo. Ningún donante falleció a los seis meses de seguimiento. En cuanto al tiempo de hospitalización posterior al procedimiento, el análisis estadístico arrojo que el promedio fue de $2(\mathrm{DE}=0,1)$ días.

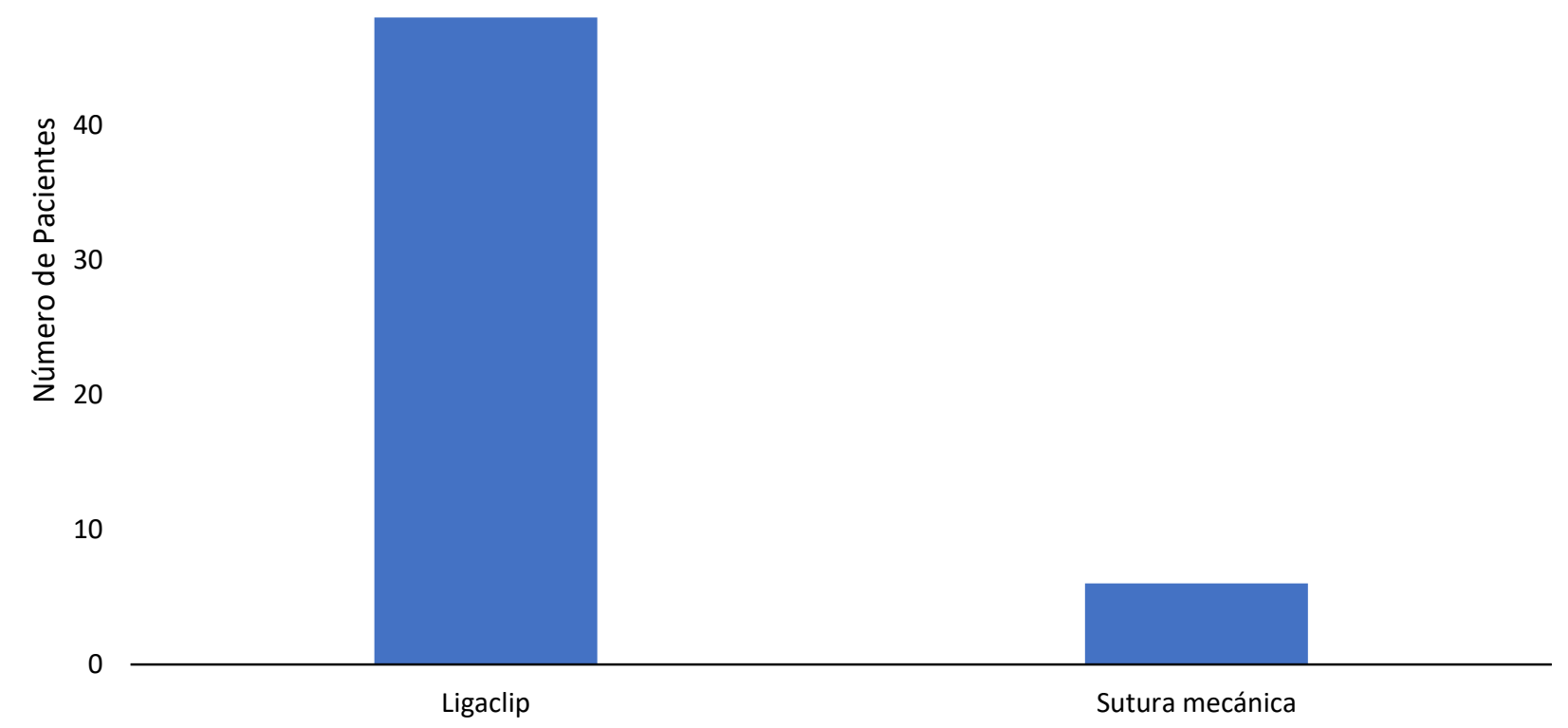

Figura 2. Gráfica de barras que muestra números de pacientes en quienes se realizó el control vascular con ligaclips de polímero no absorbible y con sutura mecánica.

\section{DISCUSIÓN}

Como lo plantea Shirodkar et $a^{19}$, los programas de donante vivo son una potencial solución al aumento permanente de las listas de espera para trasplante renal en comparación con la disponibilidad de órganos para trasplantar y es necesario entender las condiciones en las que se lleva a cabo este proceso para mejorar los resultados. Este artículo describe la experiencia de Colombiana de Trasplantes en la regional del Caribe Colombiano en su programa de donante vivo ${ }^{19}$.
De la información demográfica, se encontró que la edad promedio de los donantes fue de 41 años (rango=20-61) lo cual concuerda con reportes en Latinoamérica donde el promedio fue de 41.2 años (rango=27-60 años), por otro lado, en reportes europeos este promedio difiere levemente (promedio: 51 años) pero el rango es muy similar (rango=24-70 años). En cuanto al género que predomina en los donantes, encontramos resultados similares a los reportados por otros grupos ya que en nuestra población las mujeres 
fueron donantes en un $57,4 \%(n=31)$ de los casos; y en dichos reportes los porcentajes fueron de $62 \%$ y $50,7 \%$ respectivamente. Por otro lado, el riñón extraído con más frecuencia en este estudio fue el izquierdo en un $87 \%(n=47)$ de los casos mientras que los reportes de Aguiló et $a l^{20} \mathrm{Y}$ Wiborg et a ${ }^{21}$ son del $94,7 \%$ y el $87 \%$ respectivamente. Comparando los resultados previamente expuestos, podemos observar que la población de este estudio es similar en términos demográficos a poblaciones de donantes vivos tanto en nuestro continente como en Europa y llama la atención que como en otras series, las mujeres tienen una tendencia mayor a ser donantes para sus seres queridos ${ }^{20,21}$.

Para las variables quirúrgicas se puede observar que los resultados del equipo son muy similares o mejores que los reportados por Aguiló et $a^{20} \mathrm{y}$ Wiborg et $a l^{21}$. Como ejemplo, el tiempo quirúrgico que en el caso propio fue de 1,9 horas equivalente con el del grupo de Aguiló ${ }^{20}$ (1,9 horas) y mejor que el grupo de Wiborg ${ }^{21}$ (3,8 horas). En el caso del tiempo de isquemia caliente el tiempo de nuestro grupo de trasplantes fue de 3.1 minutos en promedio comparado con 3,2 minutos en el grupo de Wiborg ${ }^{21}$ y 6,8 minutos en el grupo de Aguiló ${ }^{20}$. La pérdida sanguínea, otra de las variables reportadas en diferentes artículos muestra que el promedio de nuestros pacientes fue de $80,5 \mathrm{ml}$ de sangre siendo casi la mitad de los reportado por el grupo de Wiborg ${ }^{21}$. Por último, es importante denotar que la tasa de conversión en las cirugías incluidas dentro de este estudio fue de cero comparada con el $4 \%$ reportado por el grupo de Aguiló ${ }^{20}$ mostrando la experticia de nuestro grupo de trasplantes en cirugía laparoscópica ${ }^{20,21}$.

Dentro de las consideraciones anatómicas del procedimiento, es claro que el número de arterias y venas renales es un aspecto importante para la ejecución del procedimiento y el estudio pretrasplante ${ }^{19}$. Por ello, se consideró relevante mostrar que en el $22 \%$ de las nefrectomías, los pacientes tuvieron arterias renales múltiples y en el $7,4 \%$ de los casos hubo más de una vena renal presente. Estas prevalencias son más valiosas al compararlas con las reportadas por diferentes grupos en las que la proporción de pacientes con arterias renales múltiples oscila entre el $8 \%$ y el $13 \%$; y en las que se puede observar que la anatomía de la población estudiada localmente muestra una mayor cantidad de pacientes con dicha variante anatómica ${ }^{20,22,23}$. Adicionalmente y teniendo en cuenta hallazgos del metaanálisis realizado por Zorgdrager et $\left.a\right|^{24}$. en el año 2016, en el que se observa una mayor tasa de complicaciones en los casos de arterias renales múltiples; podemos resaltar los resultados obtenidos ya que a pesar de tener una mayor proporción de pacientes con arterias renales múltiples, la tasa de complicaciones intraoperatorias según la clasificación de Clavien modificada para nefrectomía laparoscópica de donante vivo se ha mantenido en cero ${ }^{25}$.

El uso de ligaclip de polímero no absorbible para el control vascular es un punto controversial pues existen grupos que se inclinan hacia el uso de este último de forma rutinaria y existen otros grupos que consideran que este método no es seguro y no debería ser utilizado argumentando un aumento en las complicaciones vasculares intraquirúrgicas por movilización del clip del muñón de la arteria renal ${ }^{26,27}$. En la serie de pacientes presentada, en el $88,8 \%(n=48)$ del total de los pacientes operados se utilizó ligaclips de polímero no absorbible como método de control vascular de la arteria renal y en el $100 \%$ de las NLMA izquierdas se realizó el mismo procedimiento, sin que se presentara complicación alguna durante o después de la cirugía. Estos resultados concuerdan con los reportados previamente por Girón et $a l^{28}$ y Baldwin et $a l^{29}$ en los que se argumenta que la técnica es segura, eficiente y coherente con la realidad económica de países como Colombia debido a su menor costo con respecto a las suturas mecánicas utilizadas en el medio ${ }^{28-30}$.

Los argumentos presentados previamente permiten empezar un proceso de conocer la población local en aras de diseñar soluciones viables que se acomoden a la realidad nacional y regional de la costa Caribe colombiana. Asimismo, esta información es de gran importancia ya que a pesar de similitudes importantes con lo que reportan otros grupos de trasplantes, hay particularidades que tienen que ser tenidas en cuenta para la ejecución exitosa de programas de salud y que son muy diferentes de lo ocurre en otras comunidades. 


\section{CONCLUSIÓN}

Debido a que la enfermedad renal crónica es una condición clínica que ha venido creciendo preocupantemente en Colombia y que incluso puede llegar a ser considerada como un problema de salud pública, es necesario tener información con rigor científico de las estrategias utilizadas en todo el mundo como lo es la NLMA y el trasplante de donante vivo para mitigar el impacto social y económico de esta enfermedad. Por otro lado, los resultados del procedimiento quirúrgico establecido y estandarizado en nuestro grupo de trasplantes asociado a un grupo de cirujanos de trasplantes experimentado, representa una opción segura de tratamiento para pacientes que se encuentran en diálisis. Finalmente, es necesario resaltar que en el seguimiento a los seis meses no se presentó ningún caso de muerte en el grupo de donantes.

\section{DECLARACIÓN SOBRE CONFLICTO DE INTERESES}

Los autores declaran no tener ningún conflicto de interés de tipo financiero, académico o laboral que ponga en riesgo los resultados de la investigación realizada.

\section{CONTRIBUCIÓN DE LOS AUTORES}

Primer autor: diseño, metodología, recolección de información, escritura y revisión.

Segundo autor: metodología, recolección de información, análisis estadístico, escritura.

Tercer autor: diseño, escritura y revisión.

\section{REFERENCIAS BIBLIOGRÁFICAS}

1. Ratner LE, Ciseck LJ, Moore RG, Cigarroa FG, Kaufman HS, Kavoussi LR. Laparoscopic live donor nephrectomy. Transplantation [revista en la Internet]. 1995 Nov;60(9):1047-9. Disponible en: https://www.ncbi.nlm.nih.gov/pubmed/7491 680.
2. Levin A, Tonelli M, Bonventre J, Coresh J, Donner J-A, Fogo $A B$, et al. Global kidney health 2017 and beyond: a roadmap for closing gaps in care, research, and policy. Lancet. 2017 Oct;390(10105):1888-917. Doi: https://doi.org/10.1016/S01406736(17)30788-2.

3. Wolf JSJ, Tchetgen MB, Merion RM. Handassisted laparoscopic live donor nephrectomy. Urology. 1998 Nov;52(5):8857. Doi: https://doi.org/10.1016/S00904295(98)00389-6.

4. Shohab D, Jamil I, Khan I, Khawaja M, Khan M, Akhter S. Hand-assisted laparoscopic donor nephrectomy: A single-center study. Saudi J Kidney Dis Transplant [revista en la Internet]. 2017 May 1;28(3):661-3. Disponible en: http://www.sjkdt.org/article.asp?issn=1319-

2442.

5. Alcaraz A, Rosales A, Palou J, Caffaratti Sfulcini J, Montlle $M$, Segarra J, et al. Nefrectomía laparoscópica de donante vivo para trasplante Renal. Los dos primeros años de experiencia. Arch Españoles Urol [revista en la Internet]. 2004;57:1091-8. Disponible en: https://medes.com/publication/20167.

6. Raber B, Westmoreland M, Arnold D, Derek B, Lueking $\mathrm{R}$, Lassiter $\mathrm{G}$, et al. Laparoscopic donor nephrectomy: $A$ single institution minimally invasive general surgeon experience 1999-2013. Am J Surg. 2017 Dec;214(6):1220-5.

Doi: https://doi.org/10.1016/j.amjsurg.2017.0 8.038 .

7. Fabián JF, Mancilla E, Aburto JS, Kasep J, Lopez JO, Almaguer F, et al. Hand-Assisted Laparoscopic Nephrectomy for Live Donor Kidney Transplantation. Transplant Proc [revista en la Internet]. 2016;48(2):568-571. Doi:

https://doi.org/10.1016/j.transproceed.2016. 02.020 
8. Kumar S, Witt RG. Hand-assisted laparoscopic retroperitoneal donor nephrectomy: A single- - institution experience of over 500 cases - Operative technique and clinical outcomes. Clin Transplant. 2018;32(6) e13261.

Doi: https://doi.org/10.1111/ctr.13261.

9. Wang $H$, Chen R, Li T, Peng L. Robot-assisted laparoscopic vs laparoscopic donor nephrectomy in renal transplantation: A meta-analysis. Clin Transplant. 2019 Jan;33(1):e13451.

Doi: https://doi.org/10.1111/ctr.13451.

10. Ricotta C, Cintorino D, Pagano D, Bonsignore $P$, Piazza S, di Francesco $F$, et al. Enhanced Recovery after Implementation of Surgery Protocol in Living Kidney Donors: The ISMETT Experience. Transplant Proc. 2019 Oct; Doi: https://doi.org/10.1016/j.transproceed.2019. 04.089 .

11. Achit $H$, Guillemin F, Karam G, Ladriere $M$, Baumann C, Frimat $L$, et al. Cost-effectiveness of four living-donor nephrectomy techniques from a hospital perspective. Nephrol Dial Transplant. $2019 \quad$ Aug; Doi: https://doi.org/10.1093/ndt/gfz143.

12. Hanson CS, Sautenet B, Craig JC, Chapman JR, Knoll G, Reese PP, et al. Informative for Decision Making? The Spectrum and Consistency of Outcomes After Living Kidney Donation Reported in Trials and Observational Studies. Transplantation. 2019 Feb;103(2):284-90.

Doi: https://doi.org/10.1097/TP.00000000000024 89.

13. Osorio-Arango $\mathrm{K}$, Beltrán-Durán $\mathrm{M}$, AriasMurillo $Y$, Prieto Alvarado $F$, Robayo A. Survival in renal transplant recipients in Colombia, 2008-2012. Biomédica. 2017;37(2):175-83. Doi: http://dx.doi.org/10.7705/biomedica.v37i2.3 246.
14. Instituto Nacional de Salud - Red nacional de trasplantes. Informe anual red de donación y trasplantes [Internet]. [consultado 2019 Nov 6]. Disponible en: https://www.ins.gov.co/Direcciones/RedesSal udPublica/DonacionOrganosYTejidos/Estadist icas/Informe-Anual-Red-Donacion-

Trasplantes-2018.pdf.

15. United Network for Organ Sharing (UNOS). Kidney Transplant by Donor Type: 2018. UNOS [Internet]. 2019;1-2. Disponible en: https://unos.org/data/transplant-trends/.

16. Tan JC, Gordon EJ, Dew MA, LaPointe Rudow D, Steiner RW, Woodle ES, et al. Living Donor Kidney Transplantation: Facilitating Education about Live Kidney Donation-Recommendations from a Consensus Conference. Clin J Am Soc Nephrol. 2015 Sep;10(9):1670-7. Doi: http://dx.doi.org/10.2215/CJN.01030115.

17. Murcia E. Trasplante renal y donacion: Analisis critico. Rev Ciencias la Salud. 2016;14(2):143-5. Disponible en: https://revistas.urosario.edu.co/xml/562/562 45910001/index.html.

18. Fondo Colombiano de Enfermedades de Alto Costo. Situación de la enfermedad renal crónica, la hipertensión arterial y la diabetes mellitus en Colombia [Internet]. [consultado 2019 Nov 6]. Disponible en: https://www.cuentadealtocosto.org/site/ima ges/Publicaciones/Situacion_ERC_HA_DM_Co lombia_2016.pdf.

19. Shirodkar SP, Sageshima J, Bird VG, Martinez $J M$, Chen L, Burke GW, et al. Living donor nephrectomy: University of Miami technique and current results. Arch Esp Urol. 2010 Apr;63(3):163-70. Disponible en: https://pdfs.semanticscholar.org/9b6a/029bc b79ca0d462c63a224af18a88e05cc91.pdf.

20. Aguiló M J, Matus F C, Leiva L L, Pérez C P, Castillo H F, Vergara M J, et al. Nefrectomía por laparóscopia de donante vivo. Experiencia 
de 75 casos consecutivos. Vol. 67, Revista chilena de cirugía . scielocl ; 2015. p. 57-60. Doi: $\quad$ http://dx.doi.org/10.4067/S071840262015000100009.

21. Wiborg $\mathrm{MH}$, Toft $\mathrm{A}$, Jahn $\mathrm{H}$, Hansen LU, Lund L. Initial experience with hand-assisted laparoscopic donor nephrectomy: a singlecentre experience over 5 years. Scand J Urol. $2017 \quad$ Feb;51(1):73-77. Doi: https://doi.org/10.1080/21681805.2016.1249 025.

22. Gurkan A, Kacar S, Basak K, Varilsuha C, Karaca C. Do multiple renal arteries restrict laparoscopic donor nephrectomy?. Transplant Proc. 2004;36(1):105-7. Doi: https://doi.org/10.1016/j.transproceed.2003. 11.064 .

23. Patil AB, Javali TD, Nagaraj HK, Babu SMLP, Nayak A. Laparoscopic donor nephrectomy in unusual venous anatomy - donor and recepient implications. Int Braz J Urol. 2017;43(4):671-8. Doi: https://doi.org/10.1590/S16775538.IBJU.2016.0309.

24. Zorgdrager M, Krikke $C$, Hofker SH, Leuvenink HGD, Pol RA. Multiple Renal Arteries in Kidney Transplantation: A Systematic Review and Meta-Analysis. Ann Transplant. 2016 Jul;21:469-78.

Doi: https://doi.org/10.12659/aot.898748.

25. Desai MR, Ganpule AP, Gupta R, Thimmegowda $M$. Outcome of renal transplantation with multiple versus single renal arteries after laparoscopic live donor nephrectomy: a comparative study. Urology. 2007 May;69(5):824-7. https://doi.org/10.1016/j.urology.2007.01.02 6.

26. Janki S, Verver D, Klop KWJ, Friedman AL, Peters TG, Ratner LE, et al. Vascular management during live donor nephrectomy: an online survey among transplant surgeons. Am J Transplant. 2015 Jun;15(6):1701-7. Doi: https://doi.org/10.1111/ajt.13142.

27. Cabello R, Garcia JV, Quicios C, Bueno G, Gonzalez C. Is There a New Alternative for a Safer Kidney Artery Ligation in Laparoscopic Donor Nephrectomy? J Laparoendosc Adv Surg Tech A. 2017 Jul;27(7):715-6. Doi: https://doi.org/10.1089/lap.2016.0271.

28. Giron F, Baez Y, Nino-Murcia A, Rodriguez J, Salcedo $S$. Use of nonabsorbable polymer ligaclip in hand-assisted laparoscopic nephrectomy for living donor. Transplant Proc. 2008 Apr;40(3):682-4. Doi: https://doi.org/10.1016/j.transproceed.2008. 02.027 .

29. Baldwin DD, Desai PJ, Baron PW, Berger KA, Maynes $\mathrm{L}$, Robson $\mathrm{CH}$, et al. Control of the renal artery and vein with the nonabsorbable polymer ligating clip in hand-assisted laparoscopic donor nephrectomy. Transplantation. 2005 Aug;80(3):310-3. Doi: https://doi.org/10.1097/01.tp.0000168553.6 1631.c6.

30. Pedraza NF, Garcia AE, A. AJ, Baez Y, Giron F. Vascular Control of the Renal Pedicle Using Non-Absorbable Polymer Ligating Clips in Hand-Assisted Living Donor Laparoscopic Nephrectomies. Nephrourol Mon [Internet]. 2019;11(3):e91761.

Doi: https://doi.org/10.5812/numonthly.91761. 